\title{
A Low-Cost Improvised Nerve Stimulator is Equivalent to High-Cost Muscle Stimulator for Anorectal Malformation Surgery
}

\author{
${ }^{1}$ Division of Pediatric Surgery, Department of Surgery, Cedars-Sinai \\ Medical Center, Los Angeles, California, United States \\ 2 Department of Anesthesiology and Pediatrics, Cedars-Sinai Medical \\ Center, Los Angeles, California, United States \\ ${ }^{3}$ New Hope Foundation, Beijing, China \\ ${ }^{4}$ Independent Consultant, Bethesda, Maryland, United States \\ Eur J Pediatr Surg 2013;23:25-28.
}

Scott Short ${ }^{1}$ Keith Kimble ${ }^{2}$ Shiwei Zhai ${ }^{3}$ Gregory Frykman ${ }^{4}$ Philip Frykman ${ }^{1}$

\author{
Address for correspondence and reprint requests Philip Frykman, MD \\ PhD, Division of Pediatric Surgery, Department of Surgery, \\ Cedars-Sinai Medical Center, 8635 W. Third Street, Suite 665W, Los \\ Angeles, CA 90048, United States (e-mail: frykmanp@cshs.org).
}

\begin{abstract}
Keywords

- anorectal malformation

- posterior sagittal anorectoplasty

- muscle stimulator

- resource-poor

Aim Our hypothesis is that an improvised low-cost peripheral nerve stimulator is equally effective compared with a high-cost muscle stimulator for anorectal malformation surgery.

Methods A modified MicroStim (MS) peripheral nerve stimulator was compared with the Peña Muscle Stimulator (PS2) on identification of muscle groups during surgery, tissue energy delivery, and cost. A consecutive series of 6 patients ( 5 boys) with weight 5.9 to $11 \mathrm{~kg}$ underwent posterior sagittal anorectoplasty (PSARP). The types of malformations included four prostatic fistulae, one bulbar fistula, and one vestibular fistula. In the course of each operation, both MS and PS2 devices were used to identify muscle groups in a head-to-head comparison with video documentation. The energy delivery was determined using a digital oscilloscope at settings used during the operations (\#9 on the MS and $120 \mathrm{~mA}$ on the PS2). Price quotes for each device were obtained from commercial vendors.

Results The subjective assessment of the surgical team was that both devices demonstrated equivalent utility in identifying critical muscle groups, although the MS probe was less convenient to use. There were no intraoperative complications, including tissue burns with either device, and no postoperative complications. The energy delivery of the devices was similar, $23.5 \mathrm{~mW}$ and $25.3 \mathrm{~mW}$ for the MS and PS2, respectively. The cost of the MS was US\$ 162 and the PS2 was US\$12,371.

Conclusion The MS nerve stimulator demonstrated equivalent effectiveness compared with the PS2 muscle stimulator at 75-fold lower cost. The MS device may serve as a low-cost effective tool for PSARP.
\end{abstract}

\section{Introduction}

Repair of anorectal malformations (ARM) using either posterior sagittal anorectoplasty (PSARP) or Georgeson's laparo- scopic technique are optimally performed using a muscle stimulator to clearly delineate the anal and pelvic muscle complexes for precise anatomic placement of the rectal pullthrough segment. ${ }^{1-3}$ Unfortunately, purchase of received

May 18, 2012

accepted after revision

July 18, 2012

published online

October 25, 2012 (c) 2013 Georg Thieme Verlag KG Stuttgart · New York
DOI http://dx.doi.org/ $10.1055 / s-0032-1326956$. ISSN 0939-7248. 


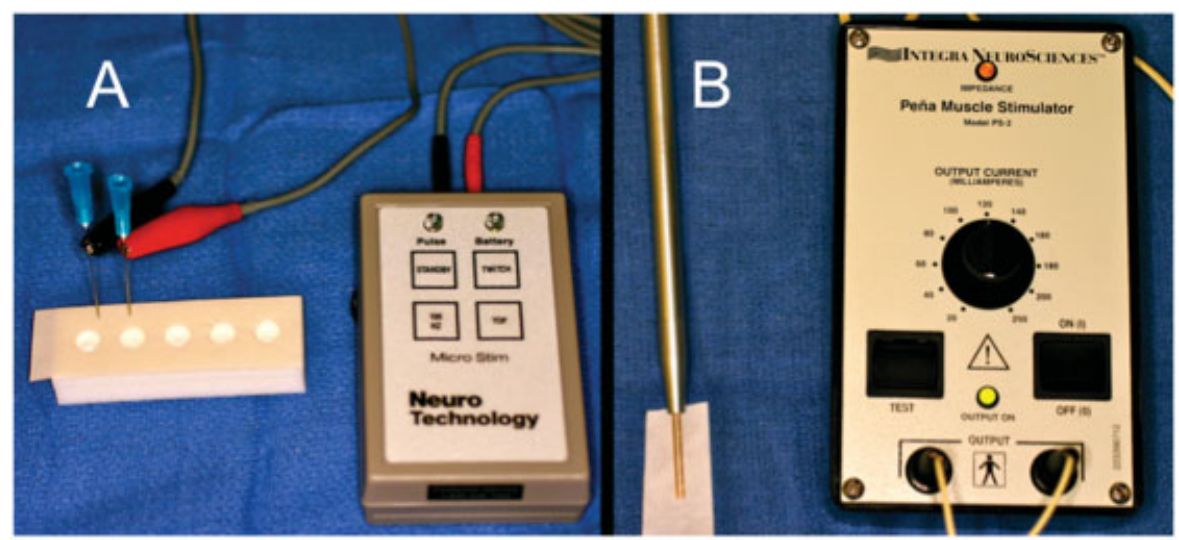

Figure 1 Stimulator devices used in this study. (A) The improvised MicroStim peripheral nerve stimulator was modified by attaching 22-gauge hypodermic needles to the alligator clips. The needles were used to contact the tissue and delineate muscle complexes during PSARP. (B) Peña muscle stimulator (PS2) and probe used for repair of anorectal malformations.

commercially available muscle stimulators for ARM surgery can be prohibitive ${ }^{4}$ for many regions of the globe due to their high cost which may exceed US $\$ 10,000$. Not surprisingly, this cost barrier limits the use of this critically important tool by surgeons in communities with limited resources to purchase this device.

Multiple authors have published an array of cleverly improvised devices to circumvent this problem, ${ }^{5,6}$ however no study has compared such devices to what might be considered a "gold standard" muscle stimulator device. Our goal was to compare a low-cost, improvised device with the PS2 Peña Muscle Stimulator on intraoperative functionality, energy delivery, and cost.

\section{Methods}

A consecutive series of patients (five boys, one girl) with weight 5.9 to $11 \mathrm{~kg}$ underwent PSARP for four prostatic fistulae, one bulbar fistula, and one vestibular fistula, respectively. Following approval from the research ethics committee, we compared use of the MicroStim (MS) peripheral nerve stimulator (Neurotechology, Houston, TX, USA), commonly used in anesthesiology, and the Peña Muscle Stimulator (PS2, Integra NeuroSciences) to identify muscle groups in a headto-head comparison with video documentation during surgery ( - Fig. 1 ). The sterile MS alligator clips were attached to two, 22-gauge hypodermic needles and the anesthesiologist away from the operative field controlled the device settings. A short-acting neuromuscular blocking agent was administered only during induction of anesthesia, and its effects were no longer present at the start of the operations. Before muscle stimulation, the tissue was moistened with warm saline in all cases, and the MS device was used in tetanic mode. The surgical team identified each muscle complex, and subjectively assessed stimulation response and ease of probe handling and recorded their impressions at the time of each operation. The video footage of each operation was subsequently reviewed retrospectively. ( - Fig. 1 )

Electrical potential, current, and energy delivery of each device were studied using a $1000 \mathrm{ohm}(1 \mathrm{k})$ resistor on a digital oscilloscope. The generated waveform, frequency, current, potential, and duty cycle were measured and recorded; the energy delivery was calculated. The MS device was tested at settings \#3 through \#10 in tetanic mode; the PS2 was tested at currents of 60 to $140 \mathrm{~mA}(\mathrm{~mA})$ as documented on the device. Device costs in USD were obtained from United State vendors.

\section{Results}

All members of the surgical team (P.F., S.Z., S.S.) uniformly felt that both devices demonstrated equivalent utility in clearly identifying all pelvic and anal muscle groups necessary to perform PSARP operations safely and effectively ( - Fig. 2). However, the team found that the improvised MS tissue contact probe was less convenient to use. There were no intraoperative complications, including tissue burns with either device, and no early or late postoperative complications. Follow-up at 5 months after PSARP, and 2 to 3 months after colostomy closure, finds that all of the children are stooling daily with a bowel management regimen of daily oral senna-based laxative. (-Fig. 2)

The properties and energy delivery of each device were compared over a range of settings in - Table 1 . Both devices delivered a square wave of $200 \mu$ s duration with frequencies of $100 \mathrm{Hertz}(\mathrm{Hz})$ and $50 \mathrm{~Hz}$ for the MS and PS2, respectively. On the basis of our patients' size, we found the optimal settings of \#9 for MS and $120 \mathrm{~mA}$ for the PS2. Not surprisingly, at these settings energy delivery of the devices was similar, 23.5 milliwatts $(\mathrm{mW})$ and $25.3 \mathrm{~mW}$ for the MS and PS2, respectively. Costs provided by United States vendors were US $\$ 162$ for MS and US\$12,371 for PS2 ( - Table 1 ).

\section{Discussion}

This study is the first to directly compare clinical use and energy delivery of an improvised stimulator device to the Peña muscle stimulator for repair of ARMs in children. The most striking finding of the study was a 75-fold lower cost of the MS compared with the PS2 with equivalent clinical 


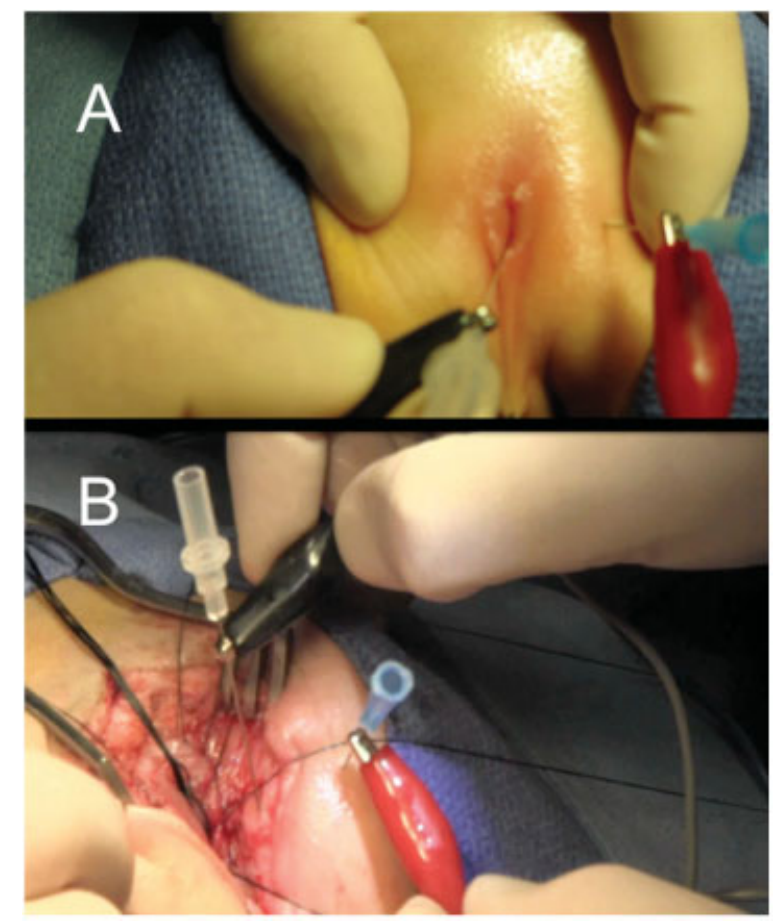

Figure 2 Application of improvised MS stimulator device. (A) Prior to repair of anorectal malformation. (B) Intraoperative delineation of muscle complex.

effectiveness. While we found that the improvised MS "probe" was less convenient to use than the PS2, the team adjusted quickly to using it.

We found that advantages of the PS2 were that the tissue contact probe was easy to use and energy delivery was available over a wide range, thus enabling use from infants to older children. However, the primary disadvantage was high cost, with a less obvious drawback being the need for the probe to be gas sterilized, a process that may not be readily available in resource-limited environments. On the other hand, the primary advantage of the improvised MS device was low cost, making it considerably more accessible either through purchase or donation. One drawback of the MS stimulator we found was that the optimal setting of energy output was near the upper limit of energy delivery for the device. Therefore, one potential concern is that the MS device may not be practical for older (larger) children where increased energy delivery would be required.

Experienced surgeons have pointed out the difficulties of obtaining critical equipment needed for treatment of ARM's in resource-limited areas of the world. ${ }^{6}$ Not surprisingly, lack of financial resources represents a key bottleneck to obtaining such instrumentation. Further highlighting the cost issue is the fact that the price of the PS2 in our study is $\sim 2.8$ times the mean annual Chinese salary, ${ }^{7}$ and more than 30 times the annual per capita income of Africa's poorest nations. ${ }^{8}$

To provide the highest quality care for their patients, pediatric surgeons in these challenging conditions are often forced to modify available equipment. One study references surgeons utilizing the electrocautery as a method to identify the muscle complex for repair of ARMs, ${ }^{4}$ however this approach risks damaging the sensitive muscular complex and associated morbidity. In an effort to maintain safety and excellence in care, other authors have modified stimulators to recapitulate the action of the Peña stimulator. ${ }^{6}$ Poenaru et al described the use of an anesthesia nerve stimulator as well as a home-made version of the "Peña" stimulator with use of Bic pen for identification of the muscle complexes while performing PSARP operations in Africa. ${ }^{5}$ While these authors created ingenious methods to delineate critical surgical anatomy, they had no means of assessing how similar (or different) their improvised devices were compared with a Peña stimulator.

Our study demonstrates that this low-cost improvised MS stimulator is safe and effective, and provided tissue energy delivery equivalent to a much more expensive stimulator. Nonetheless, our study was limited by the small sample size and the subjective assessment of outcome by the surgical team. Additionally, other improvised nerve/muscle stimulators may not be as safe or efficacious as the device tested here.

Table 1 Comparison of MicroStim and Peña muscle stimulator electrical properties. Both devices delivered a square wave of $200 \mu \mathrm{sec}$ duration with frequencies of 100 and $50 \mathrm{~Hz}$ for the MS and PS2, respectively. Optimal settings for patients in this study are noted in the shading

\begin{tabular}{|l|l|l|l|l|l|l|l|l|l|}
\hline \multicolumn{5}{|c|}{ MicroStim } & \multicolumn{5}{c|}{ Peña stimulator (PS2) } \\
\hline Dial setting & $\begin{array}{l}\text { Potential } \\
(\mathrm{V})\end{array}$ & $\begin{array}{l}\text { Current } \\
(\mathrm{mA})\end{array}$ & $\begin{array}{l}\text { Energy/pulse } \\
(\mathrm{m} / / \mathrm{\mu sec})\end{array}$ & $\begin{array}{l}\text { Power } \\
(\mathrm{mW})\end{array}$ & $\begin{array}{l}\text { Potential } \\
(\mathrm{V})\end{array}$ & $\begin{array}{l}\text { Current } \\
(\mathrm{mA})\end{array}$ & $\begin{array}{l}\text { Energy/pulse } \\
(\mathrm{m} / / \mu \mathrm{sec})\end{array}$ & $\begin{array}{l}\text { Power } \\
(\mathrm{mW})\end{array}$ \\
\hline 10 & 10 & 129 & 0.258 & 23.5 & 22 & 140 & 0.616 & 32.0 \\
\hline 9 & 10 & 129 & 0.258 & 23.5 & 20 & 120 & 0.48 & 25.3 \\
\hline 8 & 8 & 103 & 0.165 & 15.0 & 16 & 100 & 0.32 & 16.8 \\
\hline 7 & 6 & 77 & 0.092 & 8.4 & 14 & 80 & 0.22 & 11.7 \\
\hline 6 & 5 & 64 & 0.064 & 5.8 & 10 & 60 & 0.12 & 6 \\
\hline 5 & 4 & 52 & 0.042 & 3.8 & & & & \\
\hline 4 & 3.3 & 42 & 0.027 & 2.5 & & & & & \\
\hline 3 & 2.3 & 29 & 0.013 & 1.2 & & & & & \\
\hline
\end{tabular}

Abbreviations: V, volt; mA, milliampere; mJ, millijoule; $\mu s e c$, microsecond; mW, milliwatt. 
28 Improvised Nerve Stimulator Equivalent to Muscle Stimulator Short et al.

\section{Conclusion}

The improvised MS stimulator tested in this study is a safe and effective, low-cost tool for PSARP operation. The results of this study highlight the enormous unmet need for a quality, affordable stimulator device to facilitate excellent treatment of children with ARMs.

\section{Acknowledgments}

The authors would like to acknowledge the Lippey Family Endowment and the Walter and Shirley Wang Chair in Pediatric Surgery at Cedars-Sinai Medical Center, Los Angeles, California for generous support of this research.

\section{Conflict of Interest}

None

\section{References}

1 Rintala RJ, Pakarinen MP. Imperforate anus: long- and short-term outcome. Semin Pediatr Surg 2008;17(2):79-89

2 Georgeson KE, Inge TH, Albanese CT. Laparoscopically assisted anorectal pull-through for high imperforate anus-a new technique. J Pediatr Surg 2000;35(6):927-930, discussion 930-931

3 Peña A, Devries PA. Posterior sagittal anorectoplasty: important technical considerations and new applications. J Pediatr Surg 1982;17(6):796-811

4 Meier D. Opportunities and improvisations: a pediatric surgeon's suggestions for successful short-term surgical volunteer work in resource-poor areas. World J Surg 2010;34(5):941-946

5 Poenaru D, Borgstein E, Numanoglu A, Azzie G. Caring for children with colorectal disease in the context of limited resources. Semin Pediatr Surg 2010;19(2):118-127

6 Meier DE, Hesse AAJ. Anorectal Malformations. Available at: http://www.global-help.org/publications/books/help_pedsurgeryafrica77.pdf

7 [Anonymous]. China Average Salaries and Expenditures. Available at: http://www.worldsalaries.org/china.shtml

8 Eyisi N. Dissecting Africa's latest GDP per capita numbers-An unconventional look. In: Africa Policy, Global. 2011 